\title{
CONSIDERATIONS ON CYCLING IN THE CASE OF LINEAR PROGRAMMING PROBLEM (LPP)
}

\author{
Vasile CĂRUȚAȘU \\ "Nicolae Bălcescu" Land Forces Academy, Sibiu, Romania \\ v.carutasu@yahoo.com
}

\begin{abstract}
Ever since the onset of algorithms for determining the optimal solution or solutions for a linear programming problem (LPP), the question of the possibility of occurrence of cycling when one or other of these algorithms are applied was born. Thus, the fundamental question regarding this issue is under what conditions the cyclic phenomenon appears for a problem of linear programming and how to construct examples in which to do so, and as a continuation of it, which methods can be developed to avoid this phenomenon. In this study we will present some aspects regarding this issue starting from the primal simplex algorithm, by highlighting some general aspects that occur when this phenomenon happens.
\end{abstract}

Keywords: linear programming problem (LPP), simplex algorithm, cycling, basic solution, degenerated base solution

\section{Introduction}

The problem of the occurrence of the phenomenon of cycling in solving the problems of linear programming has concerned many mathematicians since the development of the simplex method by Dantzig [1].

The beginning of discussions on this subject has been shaped by the example of Hoffman [5] in which this phenomenon of cycling occurs.

Since then, the phenomenon of cycling has begun to be intensively studied, the main questions for which answers were sought being those related to:

1. The conditions of occurrence of the cycling phenomenon;

2. Creating examples of linear programming problems in which the cycling phenomenon occurs;

3. Developing rules or methods to avoid cycling phenomenon.

There are many articles that concern the phenomenon of cycling (the first question) as it can be seen in [3] or [4]. Following the example given by Hoffman, other examples of cyclic phenomena [3] or [4] have been developed. For the third question, various methods of avoiding cycling have been developed.

In [7] is presented a variant of the answer to the fundamental question, and in [3] we can find ways to build such examples.

Later, when the means of computing emerged and it was possible to make computer applications that automatically determine the solution or optimal solutions of a linear programming problem, it was found that even here the cycling phenomenon [2] or [6] can occur.

The solution to the fundamental question presented in [7] consists in solving a nonlinear inequality system, which exemplifies it in different situations.

However, in [4] there are presented issues that reveal that this situation is far from being solved, meaning that for most of the 
proposed rules and methods, examples can be found where they do not work.

In the next section we will present some concepts and clarifications that will be used in section 3 which highlight some aspects related to cycling.

\section{Basic concepts}

Let there be a linear programming problem (LPP) in the standard form:

$\left\{\begin{array}{l}\min z=c^{T} \cdot x \\ A \cdot x=b, A \in M_{m, n}(R) \\ x_{i} \geq 0,(\forall) i \in 1, n(x \geq 0)\end{array}\right.$,

where $\operatorname{rank} A=m, m \leq n$ (if the system (1) admits solutions, then it has at most $n$ linear independent equations).

We will note with

$P=\left\{x \in R^{n} / A \cdot x=b, x \geq 0\right\}$,

the set of admissible solutions or LPP programs from above.

Definition 1 Let $S=\left\{a^{1}, a^{2}, \ldots, a^{n}\right\}$ the column vectors of the matrix $A$ in the relation (1). We call base any subset consisting of $m$ linear independent vectors from the set $S$ (given that the rank $A=m$ ).

Let $B=\left\{a^{i_{1}}, a^{i_{2}}, \ldots, a^{i_{m}}\right\} \subseteq S$ a base of the space $\boldsymbol{R}^{m}$. We will note with

$J_{B}=\left\{i \in \overline{1, n} / a^{i} \in B\right\}$ (basic vectors);

$J_{R}=\left\{j \in \overline{1, n} / a^{j} \notin B\right\}$ (non-basic vectors).

Definition 2 It is called the basic solution of a LPP a vector $x \in P$ for which $x_{i}>0,(\forall)$ $i \in J_{B}$.

Definition 3 If a basic solution has exactly $m$ non-zero components, then we say we have an non-degenerate solution. If we have less than $m$ non-zero components we say we have a basic degenerate solution.

Observation 1 In other words, a vector $x \in P$ is a basic non-degenerate basic solution if $x_{i}>0,(\forall) i \in J_{B}$ and $x_{j}=0$, $(\forall) j \in J_{R}$ and is a basic degenerate solution if ( $\exists) i \in J_{B}$ so that $x_{i}=0$ and $x_{j}=0$, $(\forall) j \in J_{R}$.
Next, for clarification, we will exemplify the two situations that may occur.

For instance, the optimal solution for the following LPP:

$$
\left\{\begin{array}{r}
\max z=x_{1}+2 \cdot x_{2}+3 \cdot x_{3}-x_{4} \\
x_{1}+2 \cdot x_{2}+3 \cdot x_{3}=15 \\
2 \cdot x_{1}+x_{2}+5 \cdot x_{3}=20 \\
x_{1}+2 \cdot x_{2}+x_{3}+x_{4}=10 \\
x_{i} \geq 0, i=1, \ldots, 4
\end{array}\right.
$$

is $(5 / 2 ; 5 / 2 ; 5 / 2 ; 0)$ which is not degenerate because the number of components of a basic solution that may be strictly positive is maximum 3, but instead, for the following LPP:

$\left\{\begin{array}{r}\max z=x_{1}+2 \cdot x_{2}+3 \cdot x_{3}-x_{4} \\ x_{1}+2 \cdot x_{2}+4 \cdot x_{3}=16 \\ 2 \cdot x_{1}+3 \cdot x_{2}+5 \cdot x_{3}=20 \\ x_{1}+2 \cdot x_{2}+x_{3}+x_{4}=10 \\ x_{i} \geq 0, i=1, \ldots, 4\end{array}\right.$

the optimal solution is $(0 ; 0 ; 4 ; 6)$ which is degenerate because the number of strictly positive components of this admissible solution ( of $P$ ) is $2<3$.

It is known that cycling phenomenon may occur when we have a degenerate solution, but, at the same time an optimum solution can be degenerate.

Observation 2 The reason why in the primal simplex algorithm, when we choose an equal basis, all the non-basic unknowns with $0\left(x_{j}=0,(\forall) j \in J_{R}\right)$ and determine the basic unknowns (from the Cramer system left after the elimination of the non-basic unknowns), is that in this situation if the solution of the system is a basic solution $\left(x_{i} \geq 0,(\forall) i \in J_{B}\right)$, then the value of the objective function in this case is minimal, meaning that we obtain an optimal solution.

Definition $4 \mathrm{~A}$ base $B$ which satisfies the relation $\bar{x}^{-B}=B^{-1} \cdot b \geq 0$ is called primal admissible basis.

In the following section we will set out some simple results that occur when the cycling phenomenon occurs. 


\section{Remarks regarding the cycling phenomenon}

In this section, as mentioned earlier, there are some features that take place when the cycling phenomenon occurs.

To begin with, we will see a necessary condition that can lead to the cycling phenomenon.

Theorem 1 Cycling phenomena can occur only when we have a basic degenerate solution.

Demonstration: Since a cycle assumes that after a certain number of steps we have to return to the same base, it results that the value of the objective function remains unchanged relative to all the bases that are part of the cycle ( $B$ and $B^{\prime}$ are consecutive bases in that cycle).

We know that when changing the basis, the expression of the value of the objective function in relation to the new base $B^{\prime}$ is

$$
\bar{z}^{B^{\prime}}=\bar{z}^{B}-\left(z_{k}^{B}-c_{k}\right) \cdot \frac{x_{l}^{B}}{y_{l k}^{B}} .
$$

Since $z_{k}^{B}-c_{k}>0$ and $y_{l k}^{B}>0$, plus we must have $\bar{z}^{B^{\prime}}=\bar{z}^{B}$, it follows that $x_{l}^{B}=0$, namely the basic solution is degenerate.

Theorem 2 Always substitute in the current base a column corresponding to a component of the basic solution that is 0 .

Demonstration: If we assume that we are in a cycle ( $B$ and $B^{\prime}$ are consecutive bases in that cycle), it means that we have not reached the optimum solution and therefore there exists $j \in J_{R}$ for which $z_{j}^{B}-c_{j}>0$. Moreover, assuming the problem has an optimal solution, it follows that for any $j \in J_{R}$ for which $z_{j}^{B}-c_{j}>0$, there exists $i \in J_{B}$ for which $y_{i j}>0$.

Considering the reasoning in the previous sentence, it follows that $x_{l}^{B}=0$.

Therefore, the component of the basic solution corresponding to the column to leave the basis $B$ is 0 .

Remarks 1) The basic columns corresponding to basic solutions that have strictly positive values do not come out of the base (come out and enter in the base only on columns for which the components of the basic solution are 0).

2) Column ${ }^{-B}$ remains unchanged in a cycle (no matter how the pivot is chosen).

3) Practically, the same point is obtained, if we consider that the non-basic solutions are zero and the basic solutions with strictly positive values are not out of the base (otherwise the objective function would decrease and we can no longer return to the same base that it was at a previous step).

Theorem 3: Let there be $B, B^{\prime}$ and $B^{\prime \prime}$ three consecutive bases in a cycle and let there be $a^{l} \in B$, the column that leaves the basis $B$. Then column $a^{l}$ cannot return to the basis $B^{\prime \prime}$.

Demonstration: If we assume that $a^{l}, l \in J_{B}$ comes out of the basis and $a^{k}, k \in J_{R}$ enters the basis $\left(y_{l k}^{B}>0\right.$ pivot), then we have $z_{l}^{B^{\prime}}-c_{l}=z_{l}^{B}-c_{l}-\left(z_{k}^{B}-c_{k}\right) \cdot \frac{1}{y_{l k}^{B}}$.

As $z_{l}^{B}-c_{l}=0, z_{k}^{B}-c_{k}>0$ and $y_{l k}^{B}>0$, it follows that $z_{l}^{B^{\prime}}-c_{l}<0$, and therefore the column $a^{l}$ cannot reenter in the basis $B^{\prime \prime}$.

Remark A cycle assumes a number of at least 3 steps.

\section{Analysis of Hoffman's example}

In this section we will look at the example given by Hoffman to see in concrete terms under what conditions the cycle phenomenon occurs.

Hoffman proposed to illustrate the phenomenon of cycling the following LPP:

$\left\{\begin{array}{l}\max z=10 \cdot x_{1}-57 \cdot x_{2}-9 \cdot x_{3}-24 \cdot x_{4} \\ 0.5 \cdot x_{1}-5.5 \cdot x_{2}-2.5 \cdot x_{3}+9 \cdot x_{4} \leq 0 \\ 0.5 \cdot x_{1}-1.5 \cdot x_{2}-0.5 \cdot x_{3}+\quad x_{4} \leq 0 \\ x_{1}, x_{2}, x_{3}, x_{4} \geq 0\end{array}\right.$,

whose standard form is

$\left\{\begin{array}{l}-\min (-\mathrm{z})=-10 \cdot x_{1}+57 \cdot x_{2}+9 \cdot x_{3}+24 \cdot x_{4} \\ 1 / 2 \cdot x_{1}-11 / 2 \cdot x_{2}-5 / 2 \cdot x_{3}+9 \cdot x_{4}+x_{5}=0 \\ 1 / 2 \cdot x_{1}-3 / 2 \cdot x_{2}-1 / 2 \cdot x_{3}+x_{4}+x_{6}=0 \\ x_{1}, x_{2}, x_{3}, x_{4}, x_{5}, x_{6} \geq 0\end{array}\right.$

Before starting to solve Hoffman's problem, we note that it accepts the optimal 
solution $x_{1}=x_{2}=x_{3}=x_{4}=0$, and the maximum objective function is $z_{\max }=0$.

The matrix of the constraints system is

$$
A=\left(\begin{array}{rrrrrr}
a^{1} & a^{2} & a^{3} & a^{4} & a^{5} & a^{6} \\
1 / 2 & -11 / 2 & -5 / 2 & 9 & 1 & 0 \\
1 / 2 & -3 / 2 & -1 / 2 & 1 & 0 & 1
\end{array}\right) .
$$

If we choose the basis $B=\left\{a^{5}, a^{6}\right\}$, then $x_{1}=x_{2}=x_{3}=x_{4}=0$ (as non-basic unknowns), and in these conditions it results that $x_{5}=x_{6}=0$, and we can initiate the primal simplex algorithm, as it can be seen in the table below.

\begin{tabular}{|c|c|c|c|c|c|c|c|c|c|c|}
\hline \multirow[b]{2}{*}{ Step } & \multirow{2}{*}{ B } & \multirow[b]{2}{*}{$c_{B}$} & \multirow{2}{*}{$\bar{x}^{B}$} & -10 & 57 & 9 & 24 & 0 & 0 & \multirow{2}{*}{$\theta_{i}$} \\
\hline & & & & $a^{1}$ & $a^{2}$ & $a^{3}$ & $a^{4}$ & $a^{5}$ & $a^{6}$ & \\
\hline \multirow{4}{*}{ 0 } & $a^{5}$ & 0 & 0 & $1 / 2$ & $-11 / 2$ & $-5 / 2$ & 9 & 1 & 0 & $0^{*}$ \\
\hline & $a^{6}$ & 0 & 0 & $1 / 2$ & $-3 / 2$ & $-1 / 2$ & 1 & 0 & 1 & 0 \\
\hline & \multicolumn{2}{|c|}{$z_{j}$} & 0 & 0 & 0 & 0 & 0 & 0 & 0 & - \\
\hline & \multicolumn{2}{|c|}{$z_{j}-c_{j}$} & - & $10 *$ & -57 & -9 & -24 & 0 & 0 & - \\
\hline \multirow{4}{*}{1} & $a^{1}$ & -10 & 0 & 1 & -11 & -5 & 18 & 2 & 0 & - \\
\hline & $a^{6}$ & 0 & 0 & 0 & 4 & 2 & -8 & -1 & 1 & $0^{*}$ \\
\hline & \multicolumn{2}{|c|}{$z_{j}$} & 0 & -10 & 110 & 50 & -180 & -20 & 0 & - \\
\hline & $z_{j}-c_{j}$ & & - & 0 & $53 *$ & 41 & -204 & -20 & 0 & - \\
\hline \multirow{4}{*}{2} & $a^{1}$ & -10 & 0 & 1 & 0 & $1 / 2$ & -4 & $-3 / 4$ & $11 / 4$ & $0 *$ \\
\hline & $a^{2}$ & 57 & 0 & 0 & 1 & $1 / 2$ & -2 & $-1 / 4$ & $1 / 4$ & 0 \\
\hline & \multicolumn{2}{|c|}{$z_{j}$} & 0 & -10 & 57 & $47 / 2$ & -74 & $-27 / 4$ & $-53 / 4$ & - \\
\hline & \multicolumn{2}{|c|}{$z_{j}-c_{j}$} & - & 0 & 0 & $29 / 2 *$ & -98 & $-27 / 4$ & $-53 / 4$ & - \\
\hline \multirow{4}{*}{3} & $a^{3}$ & 9 & 0 & 2 & 0 & 1 & -8 & $-3 / 2$ & $11 / 2$ & - \\
\hline & $a^{2}$ & 57 & 0 & -1 & 1 & 0 & 2 & $1 / 2$ & $-5 / 2$ & $0 *$ \\
\hline & \multicolumn{2}{|c|}{$z_{j}$} & 0 & -39 & 57 & 9 & 42 & 15 & -93 & - \\
\hline & \multicolumn{2}{|c|}{$z_{j}-c_{j}$} & - & -29 & 0 & 0 & $18^{*}$ & 15 & -93 & - \\
\hline \multirow{4}{*}{4} & $a^{3}$ & 9 & 0 & -2 & 4 & 1 & 0 & $1 / 2$ & $-9 / 2$ & $0^{*}$ \\
\hline & $a^{4}$ & 24 & 0 & $-1 / 2$ & $1 / 2$ & 0 & 1 & $1 / 4$ & $-5 / 4$ & 0 \\
\hline & \multicolumn{2}{|c|}{$z_{j}$} & 0 & -21 & 48 & 9 & 24 & $21 / 2$ & $-141 / 2$ & - \\
\hline & \multicolumn{2}{|c|}{$z_{j}-c_{j}$} & - & -11 & -9 & 0 & 0 & $21 / 2^{*}$ & $-141 / 2$ & - \\
\hline \multirow{4}{*}{5} & $a^{5}$ & 0 & 0 & -4 & 8 & 2 & 0 & 1 & -9 & - \\
\hline & $a^{4}$ & 24 & 0 & $1 / 2$ & $-3 / 2$ & $-1 / 2$ & 1 & 0 & 1 & $0 *$ \\
\hline & \multicolumn{2}{|c|}{$z_{j}$} & 0 & 12 & -36 & -12 & 24 & 0 & 24 & - \\
\hline & \multicolumn{2}{|c|}{$z_{j}-c_{j}$} & - & 22 & -93 & -21 & 0 & 0 & $24 *$ & - \\
\hline \multirow{4}{*}{6} & $a^{5}$ & 0 & 0 & $1 / 2$ & $-11 / 2$ & $-5 / 2$ & 9 & 1 & 0 & \\
\hline & $a^{6}$ & 0 & 0 & $1 / 2$ & $-3 / 2$ & $-1 / 2$ & 1 & 0 & 1 & \\
\hline & \multicolumn{2}{|c|}{$z_{j}$} & 0 & & & & & & & - \\
\hline & \multicolumn{2}{|c|}{$z_{j}-c_{j}$} & - & & & & & & & - \\
\hline
\end{tabular}

Table no. 1 Simplex table for Hoffman's problem

The way in which elements $\theta_{i}$ were chosen, highlighted the phenomenon of cycling. But we still want to see what happens if we choose the other possible variants for $\theta_{i}$. We start this analysis from step 4 in which we will choose the other value for $\theta_{i}$, and we will thus pass from the basis $B_{5}=\left\{a^{3}, a^{4}\right\}$ to basis $B_{6}=\left\{a^{3}, a^{5}\right\}$, instead of the basis chosen previously $B_{6}=\left\{a^{5}, a^{4}\right\}$. 
Table no. 2 Simplex table for Hoffman's problem - variant 1

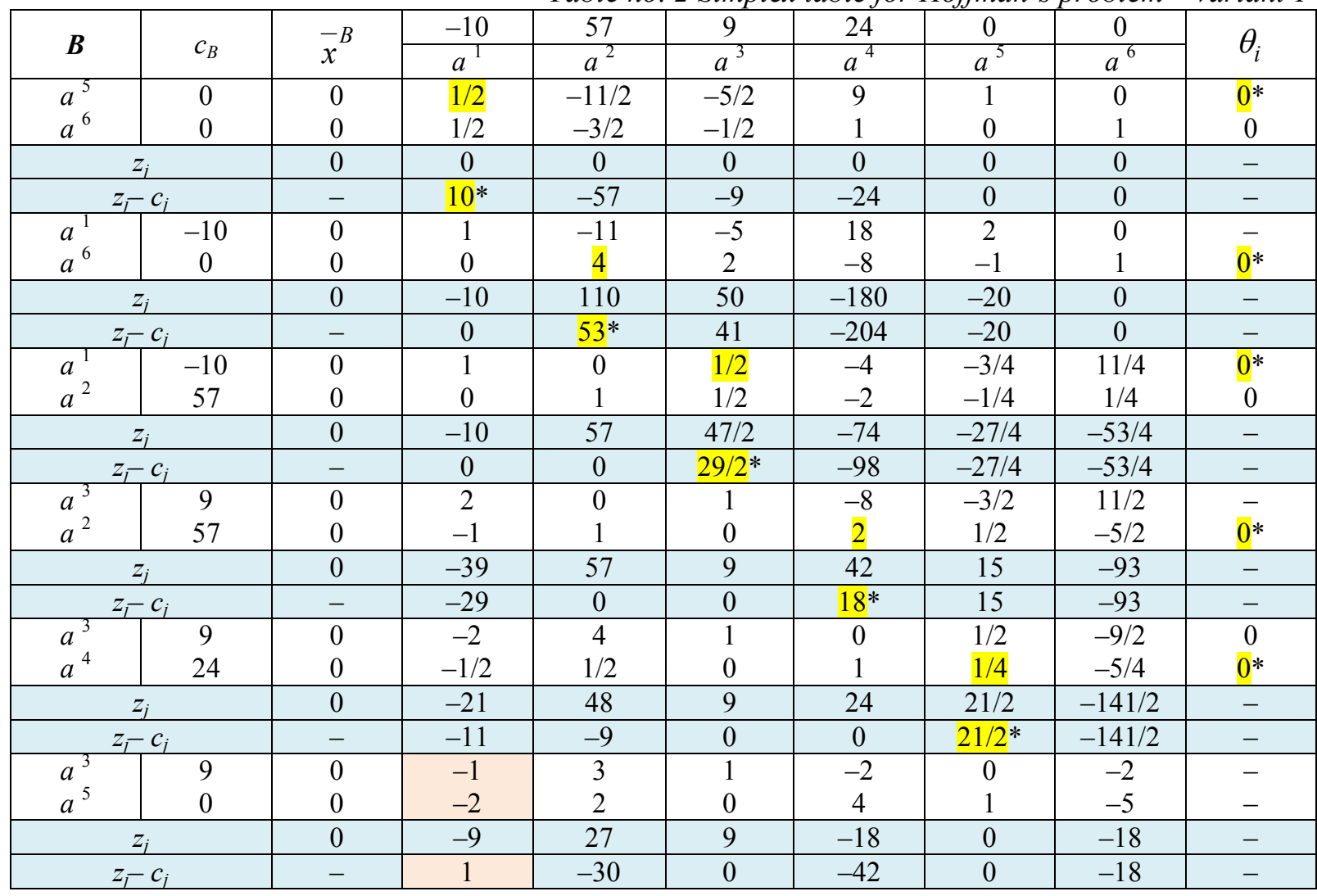

By making this choice, we see that the algorithm stops, and the conclusion that can be drawn is that the problem is optimally infinite, which cannot happens because we know that this problem admits, as we said at the beginning, an optimal solution.
We continue the analysis with the choice of the alternative value $\theta_{i}$ in Table no. 1 corresponding to the second stage in which we move from the basis $B_{3}=\left\{a^{1}, a^{2}\right\}$ to the basis $B_{4}=\left\{a^{1}, a^{3}\right\}$, instead of the base initially chosen $B_{4}=\left\{a^{3}, a^{2}\right\}$.

Table no. 3 Simplex table for Hoffman's problem - variant 2

\begin{tabular}{|c|c|c|c|c|c|c|c|c|c|}
\hline \multirow{2}{*}{$\boldsymbol{B}$} & \multirow[b]{2}{*}{$c_{B}$} & \multirow{2}{*}{$\bar{x}^{B}$} & -10 & 57 & 9 & 24 & 0 & 0 & \multirow{2}{*}{$\theta_{i}$} \\
\hline & & & $a^{1}$ & $a^{2}$ & $a^{3}$ & $a^{4}$ & $a^{5}$ & $a^{6}$ & \\
\hline$a^{5}$ & 0 & 0 & $1 / 2$ & $-11 / 2$ & $-5 / 2$ & 9 & 1 & 0 & $0 *$ \\
\hline$a^{6}$ & 0 & 0 & $1 / 2$ & $-3 / 2$ & $-1 / 2$ & 1 & 0 & 1 & 0 \\
\hline \multicolumn{2}{|c|}{$z_{j}$} & 0 & 0 & 0 & 0 & 0 & 0 & 0 & - \\
\hline \multicolumn{2}{|c|}{$z_{j}-c_{j}$} & - & $10 *$ & -57 & -9 & -24 & 0 & 0 & - \\
\hline$a^{1}$ & -10 & 0 & 1 & -11 & -5 & 18 & 2 & 0 & - \\
\hline$a^{6}$ & 0 & 0 & 0 & 4 & 2 & -8 & -1 & 1 & $0 *$ \\
\hline \multicolumn{2}{|c|}{$z_{j}$} & 0 & -10 & 110 & 50 & -180 & -20 & 0 & - \\
\hline \multicolumn{2}{|c|}{$z_{j}-c_{j}$} & - & 0 & $53 *$ & 41 & -204 & -20 & 0 & - \\
\hline$a^{1}$ & -10 & 0 & 1 & 0 & $1 / 2$ & -4 & $-3 / 4$ & $11 / 4$ & 0 \\
\hline$a^{2}$ & 57 & 0 & 0 & 1 & $1 / 2$ & -2 & $-1 / 4$ & $1 / 4$ & $0 *$ \\
\hline \multicolumn{2}{|c|}{$z_{j}$} & 0 & -10 & 57 & $47 / 2$ & -74 & $-27 / 4$ & $-53 / 4$ & - \\
\hline \multicolumn{2}{|c|}{$z_{\bar{J}}-c_{j}$} & - & 0 & 0 & $29 / 2^{*}$ & -98 & $-27 / 4$ & $-53 / 4$ & - \\
\hline$a^{1}$ & -10 & 0 & 1 & -1 & 0 & -2 & $-1 / 2$ & $-5 / 2$ & \\
\hline$a^{3}$ & 9 & 0 & 0 & 2 & 1 & -4 & $-1 / 2$ & $1 / 2$ & \\
\hline \multicolumn{2}{|c|}{$z_{j}$} & 0 & -10 & 28 & 9 & -16 & $1 / 2$ & $59 / 2$ & - \\
\hline \multicolumn{2}{|c|}{$z_{j}-c_{j}$} & - & 0 & -29 & 0 & -40 & $1 / 2$ & $59 / 2$ & - \\
\hline
\end{tabular}


By making this choice, we again notice that the same phenomenon occurs as before, with the same conclusion that the problem has an infinite optimal solution.

Finally, if in table 1 we choose the alternative value for the $\theta_{i}$ corresponding to the first step (step 0), in which we move from the basis $B_{1}=\left\{a^{5}, a^{6}\right\}$ to the basis $B_{2}=\left\{a^{5}, a^{1}\right\}$, instead of the base originally chosen $B_{2}=\left\{a^{1}, a^{6}\right\}$ we get

Table no. 4 Simplex table for Hoffman's problem - variant 3

\begin{tabular}{|c|c|c|c|c|c|c|c|c|c|}
\hline \multirow[b]{2}{*}{$\boldsymbol{B}$} & \multirow[b]{2}{*}{$c_{B}$} & \multirow[b]{2}{*}{$\bar{x}^{B}$} & & & & & & & \multirow[b]{2}{*}{$\theta_{i}$} \\
\hline & & & $\frac{-10}{a^{1}}$ & $\begin{array}{l}57 \\
a^{2}\end{array}$ & $\frac{9}{a^{3}}$ & $\begin{array}{l}24 \\
a^{4}\end{array}$ & $\begin{array}{c}0 \\
a^{5}\end{array}$ & $\begin{array}{c}0 \\
a^{6}\end{array}$ & \\
\hline$a^{5}$ & 0 & 0 & $1 / 2$ & $-11 / 2$ & $-5 / 2$ & 9 & 1 & 0 & 0 \\
\hline$a^{6}$ & 0 & 0 & $1 / 2$ & $-3 / 2$ & $-1 / 2$ & 1 & 0 & 1 & $0^{*}$ \\
\hline \multicolumn{2}{|c|}{$z_{j}$} & 0 & 0 & 0 & 0 & 0 & 0 & 0 & - \\
\hline \multicolumn{2}{|c|}{$z_{j}-c_{j}$} & - & $10^{*}$ & -57 & -9 & -24 & 0 & 0 & - \\
\hline$a^{5}$ & 0 & 0 & 0 & -4 & -2 & 8 & 1 & -1 & \\
\hline$a^{1}$ & -10 & 0 & 1 & -3 & -1 & 2 & 0 & 2 & \\
\hline \multicolumn{2}{|c|}{$z_{j}$} & 0 & -10 & 30 & 10 & -20 & 0 & -20 & - \\
\hline \multicolumn{2}{|c|}{$z_{j}-c_{j}$} & - & 0 & -27 & 1 & -48 & 0 & -20 & - \\
\hline
\end{tabular}

Also, in this case, we see that the algorithm stops, and the conclusion that can be drawn is that the problem has an infinite optimal. It is an anomaly, because normally in these situations when the cycling phenomenon is no longer produced, the optimal solution should have appeared.

\section{Concluding remarks}

There still remain a number of aspects of cycling that can be analyzed, even if important steps are taken in finding the conditions that lead to its occurrence.

These limitations tell us how we can improve established algorithms, but also the computing applications developed on the basis of these.

From the analysis of Hoffman's example we can see that if we deviate from the rule of building a cycle, we can get erroneous conclusions.

We consider that there are still important issues concerning this subject that need to be clarified. One of the questions would be about the moment when the cycling occurs, in other words, the cycling phenomenon occurs even with the first base or it occurs after a certain number of stages.

\section{References}

[1] Dantzig GB., Maximization of a linear function of variables subject to linear inequalities. In: Koopmans TC, editor. Activity analysis of production and allocation, New York, 1951.

[2] Gass SI., Comments on the possibility of cycling with the simplex method. Operations Research 1979; 27:848-52.

[3] Gass SI, Vinjamuri S., Cycling in linear programming problems. Computers \& Operations Research 2004; 31:303-11.

[4] Hall JAJ, McKinnon KIM., The simplest examples where the simplex method cycles and conditions where EXPAND fails to prevent cycling. Mathematical Programming 2004; 100(1):133-50.

[5] Hoffman AJ., Cycling in the simplex algorithm. Report 2974, Washington, DC: National Bureau of Standards: 1953.

[6] Kotiah TCT, Steinberg DI., On the possibility of cycling with the simplex method. Operations Research 1978; 26:374-6.

[7] Zörnig P., Systematic construction of examples for cycling in the simplex method, Computers \& Operations Research, 33 (2006), 2247-2262, Elsevier Ltd. 